\title{
Poster Exhibit 9: Improving Practice Management \& Beyond
}

Cite as: Can Urol Assoc / 2021;15(6S2):S158-60. http://dx.doi.org/10.5489/cuaj.7408

\section{UP-165}

Impact of physician assistants on patient volume at an outpatient Canadian urology clinic

Lames Misurka ${ }^{1}$, Miran Kenk', Jaime O. Herrera-Caceres ${ }^{1}$, Katherine Lajkosz $^{2}$, Dhiral Kot ${ }^{1}$, Antonio Finellit, Neil E. Fleshner ${ }^{7}$

${ }^{1}$ Surgical Oncology, University Health Network, Toronto, ON, Canada; ${ }^{2}$ Biostatistics, University Health Network, Toronto, ON, Canada

Introduction: Physician assistants (PAs) are being used to increase access to healthcare and reduce cost throughout Canada. We set out to determine if the introduction of PAs into a tertiary center urology oncology practice improved access for patients, while maintaining cost effectiveness.

Methods: We retrospectively reviewed OHIP billing codes, broken down quarterly, since the introduction of PAs into outpatient urology practice for two attending urologists at Princess Margaret Cancer Centre. Data were grouped into two separate periods - introduction of the first PA into the practice (1 PA: 2014 Q2 to 2015 Q3) and introduction of second PA (2 PA: 2016 Q4 to 2019 Q4). Data points were displayed using scatterplots demonstrating number of services provided for each quarter and period, along with the fitted regression lines. All regression analyses were conducted using Newey-West standard errors of lag 1. Cost associated with PAs are presented as Health Force Ontario recommendations for a new graduate PA hire.

Results: Upon introduction of the first and second PA, average number of visits increased by 308 and 191 patient visits, respectively. This translates into approximately 1000 additional patient visits per year per urologist. Linear regression coefficients for each time period and corresponding slope, confidence interval and p-value are displayed in Fig. 1. A trend towards statistical significance was observed within each time period and between the two periods. A mild net benefit was demonstrated in annual patient billing by a modest $\$ 9000$ per attending surgeon. Moreover, the average number of patients seen by PAs (approximately 3740) exceeds the additional patient visits created by the introduction of PAs, allowing physicians to spend more time with complex patients.

Conclusions: The introduction of PAs to outpatient urology clinics represents a relatively cost-neutral and effective way for improving patient access, while allowing physicians to dedicate more time to complex patients.

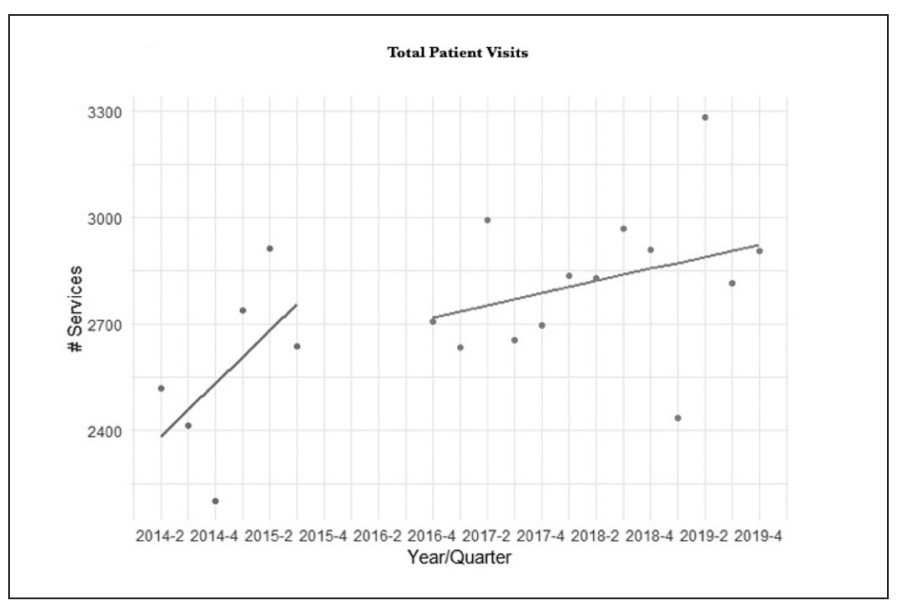

UP-165. Fig. 1. Total patient visits.

\begin{tabular}{lccc}
\hline UP-165. Table 1. & & & \\
\hline Period & Slope & $\mathbf{9 5 \%} \mathbf{C l}$ & $\mathbf{p}$ \\
\hline 1 PA & 74.9 & $(-9,158.7)$ & 0.068 \\
2 PAs & 17.4 & $(-0.5,35.3)$ & 0.056 \\
\hline
\end{tabular}

\begin{tabular}{lccc}
\hline UP-165. Table 2. & & & \\
\hline & Coefficient & $\mathbf{9 5 \%} \mathbf{C l}$ & $\mathbf{p}$ \\
$\begin{array}{l}\text { Difference in slope } \\
\text { (2 PAs - 1 PA) }\end{array}$ & -57.5 & $(-124.3,9.4)$ & 0.087 \\
\hline
\end{tabular}

\section{UP-166}

A prospective evaluation of health literacy in an outpatient prostate cancer ambulatory clinic

Karnvir S. Rai ${ }^{1}$, Oksana Harasemiw ${ }^{2}$, Uday Mann ${ }^{3}$, Navdeep Tangri ${ }^{2,4,5}$, Amanda Eng ${ }^{6}$, Jasmir G. Nayak ${ }^{3,7}$

${ }^{1}$ Rady Faculty of Health Sciences, University of Manitoba, Winnipeg, MB, Canada; ${ }^{2}$ Chronic Disease Innovation Centre, Seven Oaks General Hospital, Winnipeg, MB, Canada; ${ }^{3}$ Section of Urology, Department of Surgery, University of Manitoba, Winnipeg, MB, Canada; ${ }^{4}$ Department of Internal Medicine, University of Manitoba, Winnipeg, MB, Canada; ${ }^{5}$ Department of Community Health Sciences, University of Manitoba, Winnipeg, MB, Canada; ${ }^{6}$ Department of Family Medicine, University of Manitoba, Winnipeg, MB, Canada; ${ }^{7}$ Manitoba Prostate Centre, Cancer Care Manitoba, Winnipeg, MB, Canada

Introduction: Shared-decision making is an important element for providing patient-centered care. This is particularly important when discussing treatment options for localized prostate cancer, given the lack of consensus on an optimal strategy. Previous studies have found that prostate cancer educational materials are largely designed for people with higher literacy levels. In this study, we sought to understand and characterize the baseline health literacy levels of patients in our population undergoing investigation for prostate cancer at our centralized referral site in Manitoba.

Methods: We conducted a prospective, survey-based study of patients undergoing a prostate biopsy for the detection of prostate cancer. Male patients $>18$ years of age who were able to comprehend English, had a prostate-specific antigen (PSA) $<50 \mathrm{ng} / \mathrm{ml}$, and no prior history of prostate cancer were included. Basic demographics were collected. Health literacy was assessed using the validated Rapid Estimate of Adult Literacy-Short Form (REALM-SF), which estimates reading levels in clinical settings. Descriptive statistics were used.

Results: Between June 1 and December 31, 2020, 98 patients were included. The mean age was 67.2 years (standard deviation [SD] 8.57) with a mean PSA of $11.7 \mathrm{ng} / \mathrm{ml}$ (SD 8.27). Eighty (82.5\%) of the men were Caucasian, with $75(76.5 \%)$ patients being married. Seventeen $(17.5 \%)$ of the patients had less than a high school education, and $56(57.7 \%) \mathrm{com}-$ pleted post-secondary education. Thirty-one (31.6\%) identified as residing in a rural location. The mean REALM-SF score was 6.6/7.0, which corresponds to a $6-8^{\text {th }}$-grade reading level (will struggle with most patient education materials).

Conclusions: We identified low levels of health literacy in our population. These findings should be considered when framing discussion with patients, 
as well as when designing decision aids to best promote patient-centered care. The impact of this finding on treatment decision-making is unknown.

\section{UP-167 \\ Conflict of interest disclosures in oral presentations at major urological conferences in 2020}

Anique Le Roux', Naji J. Touma'

'Department of Urology, Queen's School of Medicine, Kingston, ON, Canada

Introduction: Reporting conflicts of interest are integral to maintaining scientific transparency. However, expectations for disclosures at conferences vary, and compliance both with guidelines and their intended spirit are often inconsistent. Our aim was to assess the frequency of disclosure slide reporting and their content at urological conferences.

Methods: We evaluated oral presentations from three major urological conferences occurring in 2020, namely the European Association of Urology conference (EAU), the American Urological Association annual meeting (AUA), and the Canadian Urological Association meeting (CUA). We collected information on disclosure slide presentation and compared it between conferences. Major categories evaluated were inclusion of a disclosure slide, number of conflicts listed, types of conflicts described, and contextualization of disclosure slide. The data was analysed using the Chi-squared test and Kruskal-Wallis test with SPSS statistical software version 26.0 .

Results: In total, 557 speakers were evaluated from the CUA ( $n=36)$, AUA $(n=160)$, and EAU ( $n=361)$. Overall, $49.01 \%$ of speakers had no disclosure slide. Among speakers with a disclosure slide, the median number of conflicts was five, median time spent on the slide was 4.38 seconds, and $30.72 \%$ gave context to disclosures. Between the different conferences, the number of speakers without a disclosure slide was $43.8 \%$ for the EAU, $61.88 \%$ for the AUA, and $44.4 \%$ for the CUA. We found that that the AUA had a lower frequency of disclosure slide reporting, while the EAU and CUA were almost identical $(p=0.001)$. There was also a significant difference for median number of conflicts listed, where EAU listed seven, AUA three, and CUA 4.5 ( $p=0.008)$.

Conclusions: Overall, disclosure slide reporting seems inconsistent across conferences. Even when reported, their value is unclear given the little time spent on them and the lack of contextualization. Explicit disclosure guidelines should be issued across urological conferences.

\section{UP-169}

Research productivity and impact of Canadian academic urologists: National trends in academic metrics as it relates to gender, subspecialty, and faculty appointment

Rebecca Power ${ }^{1}$, Jason Hearn ${ }^{1}$, Stuti M. Tanya ${ }^{1}$, Sanjay Sharma ${ }^{2}$, Ashley R. Cox ${ }^{3}$, Michael K. Organ ${ }^{4}$

${ }^{1}$ Faculty of Medicine, Memorial University of Newfoundland, St John's, NL, Canada; 'Department of Ophthalmology, Queen's University, Kingston, ON, Canada; ${ }^{3}$ Department of Urology, Dalhousie University, Halifax, NS, Canada; ${ }^{4}$ Division of Urology, Discipline of Surgery, Faculty of Medicine, Memorial University of Newfoundland, St. John's, NL, Canada

Introduction: There are multiple metrics of academic success and productivity. The objective of this study was to quantify research productivity of academic Canadian urologists on the basis of h-index, number of citations, and publications as they correlate with gender, subspecialty, and faculty appointment.

Methods: This was a cross-sectional study of academic urologists from Canadian academic institutions with a postgraduate program in urology. Academic urologists and faculty appointments were identified from uni- versity websites. Gender was determined from publicly available physician databases. $\mathrm{H}$-indices, number of citations, and publications were collected from Scopus. Descriptive, univariate, and multivariate statistics were used to analyze the relationship of h-index, number of citations, and publications with faculty appointment, subspecialty, and gender.

Results: A total of 242 academic urologists were identified. The means were $16.4 \pm 16.7$ for $\mathrm{h}$-index, $1995 \pm 402$ for number of citations, and $79 \pm 157$ for number of publications per individual. The subspeciality of uro-oncology had the highest academic metrics. Full professor appointments had significantly higher $h$-indices $(p<0.001)$. There were large discrepancies noted in gender; male urologists had significantly higher $\mathrm{h}$-indices ( $\mathrm{p}=0.006), 94.2 \%$ of urology professors were male, and the subspecialities of male fertility and transplant had no female representation in the collected database. Female reconstruction $(28.0 \%)$ and pediatrics $(27.8 \%)$ had the greatest female representation, as well as the second and third highest $\mathrm{h}$-indices, among all subspecialties.

Conclusions: There are large gender disparities in faculty appointment and academic metrics in Canadian urology. This study is the first to show that subspecialities with higher female representation maintain a high level of research productivity, as measured by h-index, number of citations, and publications.

\section{UP-170}

Gender disparity in editorial boards of major urology journals Anudari Zorigtbaatar ${ }^{1}$, Anne Xuan-Lan Nguyen ${ }^{7}$, David Bouhadana', David-Dan Nguyen ${ }^{1}$, Ashley R. Cox ${ }^{2}$, Naeem Bhojani ${ }^{3}$

${ }^{1}$ Faculty of Medicine, McGill University, Montreal, QC, Canada; ${ }^{2}$ Department of Urology, Dalhousie University, Halifax, NS, Canada; ${ }^{3}$ Division of Urology, University of Montreal Hospital Centre, Montreal, QC, Canada

Introduction: The lack of female representation in academic urology has been suggested to be one of the drivers of gender disparity in the field. Joining editorial boards (EBs) of academic journals was noted to have a lasting impact on career development. As such, gender disparities in editorial board composition may contribute to those observed in academic urology. This study aimed to evaluate the gender distribution in EBs of major urology journals.

Methods: We identified EB members of the five highest-impact urology journals based on their 2019 Impact Factor score. EB member's gender, country of practice, and academic position were collected using publicly available data. The gender was specifically determined by identifying the pronoun used in biographies. Additionally, gender was further validated using Gender-API, a gender-assigning program previously published in the literature.

Results: Female proportion of individual journals' EBs ranged from 8.6$28.9 \%$ (Table 1). In total, 307 unique current EB members were identified. Of those, $43(14.0 \%)$ were women. Almost a third of female EB members sat on more than one EB. Both men and women had the same proportion of associate professors $(23.3 \%$ and $23.1 \%$, respectively). A larger proportion of men $(39.5 \%)$ than women $(32.6 \%)$ were full professors. In addition, all female members were from high-income countries and $53.5 \%$ of them were in the U.S.

Conclusions: There is a significant disparity in the number of women sitting on EBs of urology journals. Female EB members are often sitting on multiple boards and are mostly from the U.S. Increased efforts to recruit more female researchers to sit on EBs are necessary to align the academic practice of urology to the progress in representation made in clinical practice.

UP-170. Table 1. Gender distribution of editorial boards of the five highest-impact urology journals

\begin{tabular}{lccccc}
\hline & Journal A & Journal B & Journal C & Journal D & Journal E \\
\hline Women (\%) & $10(15.9)$ & $14(10.8)$ & $7(8.6)$ & $13(15.3)$ & $11(28.9)$ \\
Men (\%) & $53(84.1)$ & $116(89.2)$ & $74(91.4)$ & $72(84.7)$ & $27(71.1)$ \\
\hline
\end{tabular}




\section{UP-171 \\ Prospective, multicenter outcome following surgical intervention for chronic scrotal content pain}

Andrew Nashed ${ }^{\prime}$, Matthew Ziegelmann ${ }^{2}$, Neel Parekh', Premal Patel ${ }^{\prime}$

${ }^{1}$ Urology, University of Manitoba, Winnipeg, MB, Canada; ${ }^{2}$ Urology,

Mayo Clinic, Rochester, MN, United States; ${ }^{3}$ Urology, Cleveland Clinic, Cleveland, $\mathrm{OH}$, United States

Introduction: Surgery for chronic scrotal content pain (CSCP) includes epididymectomy, vasectomy reversal (VR), or microscopic denervation of the spermatic cord (MDSC). Short-term followup studies demonstrate adequate pain relief and improved quality of life. We conducted a multicenter study examining outcomes in patients treated with surgery for CSCP. Our study sought to fully characterize the efficacy of surgical intervention and provide long-term data that may be used to educate patients and providers on expected outcomes following surgical management of CSCP.

Methods: We identified patients who underwent MDSC, epididymectomy, or VR for CSCP by three surgeons at separate institutions between July 2019 and January 2020. Preoperative spermatic cord block (SCB) was performed in all patients to determine surgical candidacy. Patients with a positive response to SCB ( $>50 \%$ pain reduction) are deemed candidates for surgical treatment. Preoperative and postoperative chronic orchalgia symptom index (COSI) scores, a validated instrument to assess CSCP, were obtained to assess treatment success.

Results: In total, 26 patients underwent MDSC ( $\mathrm{n}=19,73 \%)$, epididymectomy $(n=6,23 \%)$, or vasovasostomy $(n=1,4 \%)$. Median pain duration 36 months (range 3-240). Median pain improvement (percentage) with preoperative SCB was $100 \%$ (range $50-100 \%$ ), and duration of improvement was eight hours (range 5-48). At median postoperative followup of six weeks (range 4-13), the median decrease in COSI scores was 16 points (range 4-26, $\mathrm{p}<0.0001$ ), with significant improvements in all subdomains (pain, sexual symptoms, quality of life). No patient experienced testicular atrophy/loss.

Conclusions: We identified significant short-term improvements in pain, sexual symptoms, and quality of life for patients with CSCP undergoing surgical management. While this data is promising, longer-term followup is necessary to determine whether improvements persist.

\section{UP-172}

Cost analysis and feasibility of a minimally invasive, in-office hydrocelectomy

Andrew A. Di Pierdomenico' ${ }^{1}$, Jeffrey Law', Alp Sener ${ }^{1}$, Jeffrey D. Campbell ${ }^{1}$ ${ }^{1}$ Department of Surgery, Division of Urology, Western University, London, ON, Canada

Introduction: Procedures performed in the operating room (OR) have considerable costs associated with labor, materials, and facility expenditures. We sought to assess the safety and efficacy of our experience with an in-office hydrocelectomy and explore the cost savings at our institution. Methods: We prospectively collected data evaluating our experience with a "minimal-incision modified fenestration" hydrocelectomy under local anesthetic. Principles of this technique include: 1 ) ipsilateral spermatic cord block; 2) $2 \mathrm{~cm}$ transverse scrotal incision and hydrocele drainage; 3 ) mobilization then excision of the redundant tunica vaginalis; and 4) oversewing of the resection margins for hemostasis. We report on our complications and recurrence rate. An exploration into potential cost savings was performed with data provided by the Department for Quality
Measurement and Clinical Decision Support at St. Joseph's Hospital using cost data for hydrocelectomy cases performed in the operating room during the 2018-2019 fiscal year.

Results: Eight in-office hydrocelectomies were performed in seven patients (mean age: 52.5 years, range 20-81). There were no recurrences or complications observed over a mean followup of 9.2 months (range 3-12). Hydrocelectomy performed in the OR had an average estimated cost of $\$ 2223 /$ procedure. The largest component of this cost was labor at \$744/ procedure, of which the post-anesthetic care unit (\$301), OR (\$220), and day surgery units (\$157) were the largest constituents. The labor cost of an in-office hydrocelectomy ranges from $\$ 34-49 /$ procedure, which yields a labor savings of at least \$695/procedure. Reduced materials, medications, cleaning, and maintenance further improve cost savings to more than $\$ 1000 /$ procedure

Conclusions: In-office hydrocelectomy is safe and not associated with an increased risk of complications in our small cohort. Performing hydrocelectomies in an ambulatory setting may provide substantial cost savings to the hospital.

\section{UP-173}

Are we prescribing medications responsibly to nonagenarians seeking urological care?

Emily Chedrawe ${ }^{1}$, Anjali Maria Lobo ${ }^{2}$, Tarek Lawen², Ashley R. Cox ${ }^{2}$

${ }^{1}$ Faculty of Medicine, Dalhousie University, Halifax, NS, Canada; ${ }^{2}$ Department of Urology, Dalhousie University, Halifax, NS, Canada Introduction: As life expectancy continues to increase, urologists will be expected to be more comfortable managing the oldest members of our population. In 2020, there were an estimated 323069 nonagenarians in Canada. Our previous research in nonagenarians showed hematuria and lower urinary tract symptoms (LUTS), including urinary retention, was the reason for referral in $65.6 \%$ of patients. The purpose of this study is to evaluate the frequency of anticoagulation use and assess anticholinergic burden in this nonagenarian population.

Methods: We performed a retrospective chart review for nonagenarians referred to urology at Dalhousie University-affiliated hospitals from 20092014. We specifically recorded their medication lists, as well as medications prescribed at their visit. The anticholinergic burden for each patient was calculated using the Anticholinergic Cognitive Burden Scale (ACB). Results: Data was collected for 154 nonagenarians referred to urology. Hematuria was the most common reason for referral $(n=43,27.9 \%)$. Of those referred for hematuria, $78.1 \%$ were on anticoagulation therapy. Urinary retention and LUTS was seen in 22 and 36 patients, respectively. Mild, moderate, and severe ACB scores were seen in $76.6 \%, 9.33 \%$, and $14.0 \%$, respectively, for the study population. Of those with urinary retention, 21 of 22 had low ACB scores. Those referred for LUTS had statistically significant higher ACB scores than the general population $(1.44 \pm 1.34$ vs. $0.96 \pm 1.17, p=0.04)$. In total, four patients were started on anticholinergic medications for management of LUTS.

Conclusions: Our study shows many nonagenarians referred with hematuria are on anticoagulation therapy. ACB does not appear to contribute to patients with urinary retention but is significantly higher in nonagenarians with LUTS. Review of medications in nonagenarian patients is essential to provide both urological and overall health benefits. 